\title{
Complexo celulolítico produzido por duas espécies de fungos fitopatogênicos isolados de mandioca
}

\author{
Martival dos Santos Morais ${ }^{1}$; Neiva Tinti de Oliveira ${ }^{1}$ Polyanna Nunes Herculano ${ }^{2}$ Keila Aparecida Moreira ${ }^{3}$
}

${ }^{1}$ Universidade Federal de Pernambuco, UFPE, Departamento de Micologia, R. Prof. Nelson Chaves s/n, Cidade Universitária, CEP 50670-420, Recife, PE. ${ }^{2}$ Universidade Federal Rural de Pernambuco, UFRPE, Departamento de Morfologia e Fisiologia Animal, R. Dom Manuel de Medeiros, s/n, Dois irmãos, CEP 52171-900, Recife, PE. ${ }^{3}$ Universidade Federal Rural de Pernambuco, UFRPE, Departamento de Morfologia e Fisiologia Animal, Av. Bom Pastor, s/n, Boa Vista, CEP 55296-901, Garanhuns, PE.

Autor para correspondência: Martival dos Santos Morais (martivalcg@hotmail.com).

Data de chegada:19/12/2015. Aceito para publicação em:20/04/2016.

$10.1590 / 0100-5405 / 2151$

\section{RESUMO}

Morais, M.S.; Oliveira, N.T.; Herculano, P.N.; Moreira, K.A.Complexo celulolítico produzido por duas espécies de fungos fitopatogênicos isolados de mandioca. Summa Phytopathologica, v.42, n.3, p.249-253, 2016.

A partir de coletas realizadas em plantios comerciais de mandioca (Manihot esculenta Crantz) em 11 municípios do Estado da Paraíba, obtiveram-se 27 isolados dos fungos Colletotrichum gloeosporoides f. sp. manihotis Henn (Penn.) e Fusarium oxysporum Schlent, fitopatogênicos à cultura. Desses isolados foi obtida a produção enzimática do complexo celulolítico, sob fermentação submersa à $120 \mathrm{rpm}$ e $30^{\circ} \mathrm{C}$ durante 7 dias, tendo $\mathrm{CMC}$ (Carboximetilcelulose) como fonte de carbono. As atividades enzimáticas foram quantificadas por meio do grau de hidrólise de tiras de papel de filtro (FPAse) e de CMC (CMCase). Utilizou-se a liberação de Paranitrofenol para quantificação das atividades da $\beta$-glucosidase $(\beta G)$. As atividades mais elevadas observadas foram: 23,13 e $1,51 \mathrm{U} / \mathrm{ml}$ para FPAse e CMCase pelos isolados de C. gloeosporioides URM 7124 e 7125 . Para $\beta \mathrm{G}, 0,0014 \mathrm{U} / \mathrm{mL}$, pelo isolado 13.

Palavras-chave: Fusarium oxysporum, Colletotrichum gloeosporioides, FPase, Endoglucanase, Enzimas.

\section{ABSTRACT}

Morais, M.S.; Oliveira, N.T.; Herculano, P.N.; Moreira, K.A. Cellulolytic complex produced by two species of phytopathogenic fungi isolated from cassava. Summa Phytopathologica, v.42, n.3, p.249-253, 2016.

Material collected from commercial crops of cassava (Manihot esculenta Crantz) in 11 municipalities in the state of Paraíba included 27 isolates of the fungi Colletotrichum gloeosporoides f. sp. manihotis Henn (Penn.) and Fusarium oxysporum Schlent., which are pathogenic to the culture. From these isolates, cellulolytic enzyme complex production was obtained under submerged fermentation at $120 \mathrm{rpm}$ and $30{ }^{\circ} \mathrm{C}$ for 7 days, with CMC (Carboxymethyl
Cellulose) as carbon source. Enzyme activities were quantified by means of the degree of hydrolysis of strips of filter paper (FPAse) and CMC (CMCase). The release of Paranitrofenol was used to quantify the activities of $\beta$-glucosidase $(\beta G)$. The highest activities were: 23.13 and $1.51 \mathrm{U} / \mathrm{ml}$ for FPase and CMCase by the isolates of C. gloeosporioides URM 7124 and 7125. For $\beta \mathrm{G}, 0.0014 \mathrm{U} /$ $\mathrm{ml}$ by isolate 13 .

Keywords: Fusarium oxysporum, Colletotrichum gloeosporioides, FPase, Endoglucanase, enzymes.

A celulose é um polímero abundantemente encontrado na natureza $(21,25)$. Sua ampla distribuição deve-se principalmente à participação na composição das paredes celulares dos vegetais (20). Devido à sua estrutura molecular, a celulose é resistente, estável e de difícil degradação (4). Assim, nos processamentos industriais e ambientais em que seja necessária a hidrólise nos componentes vegetais, são exploradas as enzimas celulolíticas para degradação química desse polissacarídeo. Estas enzimas atuam na preparação de materiais das indústrias alimentícia, farmacêutica, de óleos essenciais, rações animais, polpa celulósica e papel, bem como na conversão da biomassa de resíduos agrícolas e industriais em produtos químicos e biocombustíveis $(27,29)$.

Segundo Lynd et al. (13) as celulases são classificadas de acordo com a sua ação catalítica e o local de atuação no substrato celulósico. O complexo celulolítico divide-se em três grupos: as endoglucanases (EnG, EC 3.2.1.4), que clivam internamente ligações glucosídicas nas regiões amorfas da fibra celulósica; as exoglucanases (ExG, EC 3.2.1.74) que atuam externamente nas fibras celulósicas e as $\beta$-glucosidases ( $\beta \mathrm{G}, \mathrm{EC} 3.2 .1 .91$ ) que hidrolisam os oligossacarídeos (celodextrinas e celobioses) resultantes das ações catalíticas das enzimas citadas anteriormente em glucose (7).

Os fungos filamentosos são produtores de enzimas hidrolíticas e apresentam relativa facilidade em sua excreção (2), tendo contribuído significativamente no campo da produção enzimática para fins industriais (6). Para suprimento das necessidades energéticas, muitos destes organismos produzem as enzimas celulolíticas, visando decompor a celulose de fontes vegetais. Todavia, somente poucos destes as produzem em quantidade significativa in vitro (28). Os fungos fitopatogênicos, devido à sua adaptação natural para invadir e parasitar a célula vegetal, apresentam a potencialidade de produzir e secretar enzimas lignocelulíticas $(3,16,19)$. Como exemplos de fungos fitopatógenos produtores das enzimas do complexo celulolítico foram estudados recentemente Fusarium graminearum, F. oxysporum e Myrothecium roridum $(10,21,25)$. Fungos fitopatogênicos da cultura da mandioca também apresentam as mesmas adaptações para realizarem os processos de infecção, especialmente para a penetração dos tecidos hospedeiros $(15,23)$.

Levando-se em consideração estas informações, este trabalho foi 
realizado com o objetivo de avaliar a produção de enzimas celulolíticas por fungos fitopatogênicos isolados de mandioca.

\section{MATERIAL E MÉTODOS}

A partir do material coletado em um levantamento de doenças realizado no Estado da Paraíba, efetuado por Morais et al. (18), obtiveram-se isolados de fungos fitopatogênicos em caules, folhas e raízes de mandioca em duas áreas denominadas I e II, localizadas em campos agrícolas comerciais de 11 municípios (Tabela 1). Cada uma das áreas continha 75 plantas que distanciavam de 5 a 10 metros uma da outra, dependendo do tamanho do plantio.

A fermentação seletiva dos fungos foi realizada em frascos de Erlenmeyers de $250 \mathrm{~mL}$, contendo $50 \mathrm{~mL}$ de meio composto de 2,0g: $\mathrm{KH}_{2} \mathrm{PO}_{2}, 1,4 \mathrm{~g}$ : $\left(\mathrm{NH}_{4}\right)_{2} \mathrm{SO}_{4}, 0,3 \mathrm{~g}: \mathrm{MgSO}_{4} \cdot 7 \mathrm{H}_{2} \mathrm{O}, 0,3 \mathrm{~g}: \mathrm{CaCl}_{2} \cdot \mathrm{H}_{2} \mathrm{O}, 5,0$ $\mathrm{g}$ : $\mathrm{NaNO}_{3}, 1 \mathrm{~mL}$ : Tween: 80 e $1 \mathrm{~mL}$ de solução de micronutrientes $(2,0$ g: $\left.\mathrm{CoCl}_{2}, 1,6 \mathrm{~g}: \mathrm{MnSO}_{4} \cdot \mathrm{H}_{2} \mathrm{O}, 1,4 \mathrm{~g}: \mathrm{ZnSO}_{4} \cdot \mathrm{H}_{2} \mathrm{O}, 0,5 \mathrm{~g}: \mathrm{FeSO}_{4} \cdot 7 \mathrm{H}_{2} \mathrm{O} / \mathrm{L}\right)$, $\mathrm{pH}$ inicial 5,0 e de carboximetilcelulose (CMC) 1\% (p/v) como única fonte de carbono. Os frascos e seu conteúdo foram esterilizados a 121 ${ }^{\circ} \mathrm{C}$ durante 15 minutos em autoclave. Em seguida foram preparadas suspensões de $10^{6}$ conídios $/ \mathrm{mL}$ de cada isolado (culturas com 8 dias de crescimento em meio BDA a $25^{\circ} \mathrm{C}$ e fotoperíodo de 12 horas). Posteriormente os frascos de Erlenmeyers foram inoculados com as suspensões e acondicionados em agitador orbital, na temperatura de $30{ }^{\circ} \mathrm{C}$ e $120 \mathrm{rpm}$ durante 7 dias. $\mathrm{O}$ ensaio foi realizado em duplicata.
O extrato enzimático bruto foi obtido do meio fermentado, após filtração e centrifugação a $9.000 \mathrm{~g}, 4{ }^{\circ} \mathrm{C}$, durante 15 minutos para separação da biomassa e do sobrenadante. Em seguida, o sobrenadante foi acondicionado em tubos e congelados a temperatura de $-20^{\circ} \mathrm{C}$ para as determinações analíticas.

O teor de proteínas totais foi determinado empregando-se o método de Bradford (5), utilizando-se albumina sérica bovina como proteína padrão da curva de calibração.

As atividades FPAse (celulase total) e CMCase (endoglucanases) foram determinadas de acordo com o método proposto por Ghose (9). Para determinação da FPAse, Tiras de papel de filtro Whatman ${ }^{\circ} 1$ (50 $\mathrm{mg}, 1 \times 6 \mathrm{~cm}$ ), recortadas e enroladas foram mergulhadas em solução de $0,5 \mathrm{~mL}$ do extrato enzimático com $1,0 \mathrm{~mL}$ de tampão citrato $(50$ $\mathrm{mM}, \mathrm{pH} 5,0$ ) contida em tubos de ensaio. Em seguida, o material foi incubado a $50{ }^{\circ} \mathrm{C}$ por 60 minutos.

Para determinação da CMCase, foram incubados $0,5 \mathrm{~mL}$ do extrato enzimático com $0,5 \mathrm{~mL}$ da solução de $\mathrm{CMC}$ a $1 \%(\mathrm{p} / \mathrm{v})$ em tampão citrato $(50 \mathrm{mM}, \mathrm{pH} 5,0)$. Posteriormente, esta solução foi incubada a $50{ }^{\circ} \mathrm{C}$ durante 30 minutos em banho termostatizado. A hidrólise foi interrompida com a adição de $1 \mathrm{~mL}$ do reagente Ácido Dinitrossalicílico (DNSA) e seguiu-se a quantificação do conteúdo de açúcares redutores.

A absorbância das soluções contendo os açúcares foi mensurada a $540 \mathrm{~nm}$ de comprimento de ondas, por meio de espectrofotômetro e por confrontação da leitura óptica com uma curva de calibração, na qual a D-glucose foi utilizada como padrão.

Para a determinação da $\beta \mathrm{G}$, seguiu-se a metodologia descrita por

Tabela 1. Fungos isolados de tecidos infectados de mandioca em duas áreas agrícolas de 11 municípios do Estado da Paraíba.

\begin{tabular}{|c|c|c|c|}
\hline Isolado & Espécie & Município & Localização \\
\hline 1 & Colletotrichum gloeosporioides & Lagoa Nova I & S: $07^{\circ} 03^{\prime} 12^{\prime \prime} / \mathrm{O}: 35^{\circ} 45^{\prime} 55^{\prime \prime}$ \\
\hline 2 & Colletotrichum gloeosporioides & Lagoa Nova II & S: $07^{\circ} 03^{\prime} 14^{\prime \prime} / \mathrm{O}: 35^{\circ} 45^{\prime} 56^{\prime \prime}$ \\
\hline 3 & Colletotrichum gloeosporioides & S.Miguel Taipú II & S: $07^{\circ} 04^{\prime} 51^{\prime \prime} / \mathrm{O}: 35^{\circ} 43^{\prime} 42^{\prime \prime}$ \\
\hline 4 & Fusarium oxysporum & Imaculada II & S: $07^{\circ} 21^{\prime} 52^{\prime \prime} / \mathrm{O}: 37^{\circ} 29^{\prime} 21^{\prime \prime}$ \\
\hline 5 & Colletotrichum gloeosporioides & Matinhas II & S: $07^{\circ} 06^{\prime} 18^{\prime \prime} / \mathrm{O}: 35^{\circ} 43^{\prime} 43^{\prime \prime}$ \\
\hline 6 & Colletotrichum gloeosporioides & Pilões I & S: $06^{\circ} 51^{\prime} 56^{\prime \prime} / \mathrm{O}: 35^{\circ} 37^{\prime} 47^{\prime \prime}$ \\
\hline 7 & Colletotrichum gloeosporioides URM 7081 & Pocinhos II & S: $07^{\circ} 08^{\prime} 35^{\prime \prime} / \mathrm{O}: 36^{\circ} 02^{\prime} 97^{\prime \prime}$ \\
\hline 8 & Fusarium oxysporum URM 7083 & Imaculada II & S: $07^{\circ} 21^{\prime} 52^{\prime \prime} / \mathrm{O}: 37^{\circ} 29^{\prime} 21^{\prime \prime}$ \\
\hline 9 & Colletotrichum gloeosporioides & Sapé I & S: $07^{\circ} 05^{\prime} 11^{\prime \prime} / \mathrm{O}: 35^{\circ} 14^{\prime} 49^{\prime \prime}$ \\
\hline 10 & Colletotrichum gloeosporioides & Cuité II & S: $06^{\circ} 27^{\prime} 98^{\prime \prime} / \mathrm{O}: 36^{\circ} 10^{\prime} 27^{\prime \prime}$ \\
\hline 11 & Colletotrichum gloeosporioides & Princesa Isabel I & S: $07^{\circ} 45^{\prime} 16^{\prime \prime} / \mathrm{O}: 37^{\circ} 57^{\prime} 42^{\prime \prime}$ \\
\hline 12 & Colletotrichum gloeosporioides & Pilões II & S: $06^{\circ} 51^{\prime} 53^{\prime \prime} / \mathrm{O}: 35^{\circ} 37^{\prime} 48^{\prime \prime}$ \\
\hline 13 & Colletotrichum gloeosporioides & Pocinhos I & S: $07^{\circ} 07^{\prime} 22^{\prime \prime} / \mathrm{O}: 36^{\circ} 02^{\prime} 93^{\prime \prime}$ \\
\hline 14 & Colletotrichum gloeosporioides & Teixeira II & S: $07^{\circ} 16^{\prime} 78^{\prime \prime} / \mathrm{O}: 37^{\circ} 19^{\prime} 51^{\prime \prime}$ \\
\hline 15 & Fusarium oxysporum & Princesa Isabel I & S: $07^{\circ} 44^{\prime} 86^{\prime \prime} / \mathrm{O}: 37^{\circ} 57^{\prime} 23^{\prime \prime}$ \\
\hline 16 & Colletotrichum gloeosporioides & Pocinhos I & S: $07^{\circ} 07^{\prime} 22^{\prime \prime} / \mathrm{O}: 36^{\circ} 02^{\prime} 93^{\prime \prime}$ \\
\hline 17 & Fusarium oxysporum & Princesa Isabel I & S: $07^{\circ} 44^{\prime} 86^{\prime \prime} / \mathrm{O}: 37^{\circ} 57^{\prime} 23^{\prime \prime}$ \\
\hline 18 & Colletotrchum gloeosporioides & Matinhas I & S: $07^{\circ} 06^{\prime} 17^{\prime \prime} / \mathrm{O}: 35^{\circ} 48^{\prime} 42^{\prime \prime}$ \\
\hline 19 & Fusarium oxysporum & Princesa Isabel II & S: $07^{\circ} 44^{\prime} 86^{\prime \prime} / \mathrm{O}: 37^{\circ} 57^{\prime} 23^{\prime \prime}$ \\
\hline 20 & Colletotrichum gloeosporioides & Cuité I & S: $06^{\circ} 27^{\prime} 96^{\prime \prime} / \mathrm{O}: 36^{\circ} 10^{\prime} 13^{\prime \prime}$ \\
\hline 21 & Colletotrichum gloeosporioides & Nova Floresta II & S: $06^{\circ} 27^{\prime} 12^{\prime \prime} / \mathrm{O}: 36^{\circ} 13^{\prime} 43^{\prime \prime}$ \\
\hline 22 & Colletotrichum gloeosporioides URM 7125 & Areia I & S: $07^{\circ} 01^{\prime} 41^{\prime \prime} / \mathrm{O}: 35^{\circ} 45^{\prime} 39^{\prime \prime}$ \\
\hline 23 & Colletotrichum gloeosporioides URM 7124 & Areia II & S: $07^{\circ} 02^{\prime} 65^{\prime \prime} / \mathrm{O}: 35^{\circ} 37^{\prime} 73^{\prime \prime}$ \\
\hline 24 & Fusarium oxysporum URM 7082 & Imaculada I & S: $07^{\circ} 21^{\prime} 56^{\prime \prime} / \mathrm{O}: 37^{\circ} 29^{\prime} 37^{\prime \prime}$ \\
\hline 25 & Fusarium oxysporum & Imaculada I & S: $07^{\circ} 21^{\prime} 56^{\prime \prime} / \mathrm{O}: 37^{\circ} 29^{\prime} 37^{\prime \prime}$ \\
\hline 26 & Colletotrichum gloeosporioides URM 7080 & Nova Floresta I & S: $06^{\circ} 27^{\prime} 11^{\prime \prime} / \mathrm{O}: 36^{\circ} 13^{\prime} 42^{\prime \prime}$ \\
\hline 27 & Colletotrichum gloeosporioides & Pocinhos II & S: $07^{\circ} 08^{\prime} 36^{\prime \prime} / \mathrm{O}: 36^{\circ} 02^{\prime} 92^{\prime \prime}$ \\
\hline
\end{tabular}


Deshpande \& Eriksson (8) modificada: $200 \mu \mathrm{L}$ do extrato enzimático foram adicionados a uma solução de $300 \mu \mathrm{L}$ de $\rho$-nitrofenil- $\beta$-Dglucopiranosídeo (PNPG) diluído em tampão acetato de sódio (50 $\mathrm{mM}, \mathrm{pH} 5,0)$. A mistura foi incubada a $50{ }^{\circ} \mathrm{C}$ por 30 minutos banho termostatizado e em seguida, foram adicionados $500 \mu \mathrm{L}$ de solução de carbonato de sódio $(1 \mathrm{M})$, para interrupção da reação. $\mathrm{O} \rho$-nitrofenol liberado foi quantificado espectrofotometricamente a $405 \mathrm{~nm}$. Os dados obtidos a partir da leitura das absorbâncias foram calculados segundo curva padrão de $\rho$-nitrofenol.

Uma Unidade (U) de atividade enzimática foi definida como a quantidade de enzima necessária para liberar um mmol de glucose por minuto.

A análise estatística dos dados foi processada através da análise de variância e teste Scott-Knott para as médias das atividades totais $(\mathrm{U} / \mathrm{ml})$ obtidas. O programa estatístico empregado foi o Sisvar versão 2008.

\section{RESULTADOS E DISCUSSÃO}

A variação da atividade celulolítica entre os isolados fúngicos foi significativa e em todos os casos houve atividades mesmo que em baixos índices (Tabela 2). A divergência significativa entre as atividades enzimáticas dentro de uma espécie permite diferenciar isolados dentro desse âmbito taxonômico $(11,24)$. Lima Filho et al. (11) avaliando a atividade da FPAse e de outras enzimas provenientes de isolados de Colletotrichum sp. fitopatogênicos de frutíferas, verificaram valores pouco superiores. Essas atividades também variaram de acordo com os isolados de cada vegetal: 4,89 U/mL em isolados de manga, 4,46 U/ $\mathrm{mL}$ em isolados de mamão, 4,04 U/mL em isolados de caju.

Ainda de acordo com a Tabela 2, foi atingida maior atividade $(23,13$ $\mathrm{U} / \mathrm{mL}$ ) para FPAse a partir do extrato enzimático obtido do isolado 23 de C. gloeosporioides URM 7124. Para esse mesmo isolado obteve-se a atividade de 1,20 U/mL da enzima endoglucanase (CMCase), valor que seguiu imediatamente o isolado 22 , em que observou-se o valor mais elevado para esta mesma enzima. Anand et al. (1) utilizando pectina e carboximetilcelulose como fontes de carbono, constataram que a atividade FPAse de isolados de C. capsici aumentava de acordo com a virulência da linhagem e com a idade da cultura, alcançando valores entre 9,00 e 12,00 U/mL, mas somente após 20 dias de cultivo. Em comparação a estas atividades, dois fungos isolados da mandioca foram superiores numa fração menor de tempo (sete dias de fermentação): além de C. gloeosporioides URM 7124, o isolado 11, com 13,21 U/mL.

Em estudos utilizando-se a espécie Trichoderma reesei, tendo espiga de milho triturada como fonte de carbono, o valor máximo alcançado foi de $5,20 \mathrm{U} / \mathrm{mL}$ (12). Este índice correspondeu às atividades de quatro isolados fúngicos do presente trabalho (O isolado 12, C. gloeosporioides URM 7080, isolados 26 e 27).

Em relação à CMCase, o isolado 22 de C. gloeosporioides URM

Tabela 2. Atividade celulolítica $(\mathrm{U} / \mathrm{ml})$ de Fusarium oxysporum e Colletotrichum gloeosporioides fitopatogênicos a mandioca

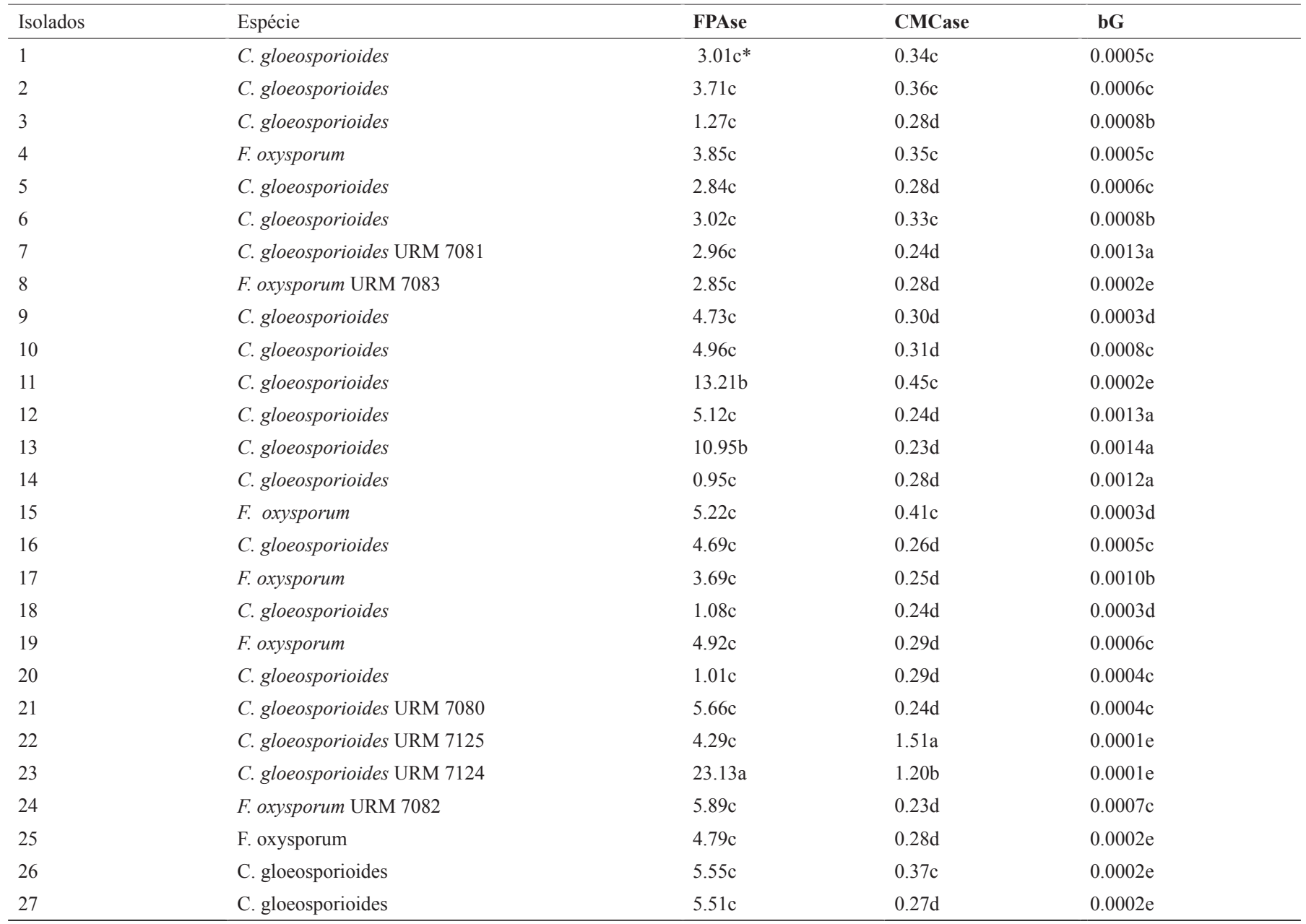

* médias seguidas da mesma letra não diferem entre si pelo teste de Scott-Knott a $5 \%$ de probabilidade 
7125, foi o fungo que apresentou a mais elevada atividade, $1,51 \mathrm{U} / \mathrm{mL}$. Em fungos saprofíticos se observam variadas taxas de atividades para esta enzima: Sukumaran et al. (28) obtiveram 14,98 U/mL de CMCase utilizando Trichoderma reesei RUT C30. Enquanto Malik et al. (14) obteveram o máximo de produção 1,57 para T. viride, em fermentação submersa pelo período de incubação de $72 \mathrm{~h}$ a $30^{\circ} \mathrm{C}$ e ajustando-se o $\mathrm{pH}$ para 5,5, alcançou-se a máxima produção de 1,66 U/mL. Estes valores são equivalentes aos alcançados no presente trabalho.

A mais elevada atividade para $\beta \mathrm{G}$ foi apresentada pelo isolado 13, C. gloeosporioides, $(0,0014 \mathrm{U} / \mathrm{mL})$, a qual não diferenciou estatisticamente dos isolados 7, 12 e 14. Esta taxa foi próxima de valores atingidos por fungos da mesma espécie fitopatogênicos à seringueira (Hevea brasiliensis), os quais apresentaram atividades 0,030 e $0,014 \mathrm{U} / \mathrm{mL}$, mesmo assim, após 10 dias em fermentação contendo carboximetilcelulose como fonte de carbono (26).

Embora o gênero Colletotrichum seja produtor desta enzima, baixas atividades podem ser previsíveis, uma vez que a maioria das espécies fúngicas a produzem intracelularmente e com pequena atividade extracelular. Este fenômeno é observado tanto para espécies de fungos filamentosos como para bactérias (17) e que mesmo em espécies mais exploradas para produção de celulases industriais, como T. reesei, sua secreção in vitro é insuficiente $(22,30)$.

Em relação à espécie $F$. oxysporum, o isolado 24,URM 7082 apresentou o maior índice de atividade para FPAse, 5,89 U/mL. A esse valor seguiu-se a atividade do isolado 15 , com 5,22 U/mL para FPAse e 0,41 para CMCase. Para a atividade da FPAse, o valor quantificado não diferiu estatisticamente dos isolado 24. (Tabela 2). Estes isolados apresentaram moderada produção de enzimas, sendo o valor da FPAse superior aos de isolados da mesma espécie, patogênicos ao tomateiro, para os quais, o mais elevado valor foi de $1,43 \mathrm{U} / \mathrm{mL}$, sendo necessário um período de fermentação de 12 dias à temperatura de $50{ }^{\circ} \mathrm{C}, \mathrm{pH}$ 6.0 (25). Este resultado difere do presente trabalho, no qual se obteve superiores resultados no período de 7 dias. Para CMCase, Kikot et al. (10) obtiveram $0,33 \mathrm{U} / \mathrm{mL}$ como maiores valores de atividade para $F$. graminearum, no quarto dia de incubação. Estas observações confirmam as conclusões de vários estudos do potencial celulolítico por fungos integrantes deste gênero.

Em vista destes resultados, foi confirmado o potencial biotecnológico dos fungos estudados para produção de celulases, sugerindo a necessidade de estudos futuros de caracterização e otimização das condições de produção destas e de outras enzimas, com possibilidade de exploração em benefício do meio ambiente e contribuição para a indústria biotecnológica.

\section{Agradecimentos}

Os autores agradecem à Coordenação do Laboratório de Biotecnologia da Universidade Federal Rural de Pernambuco, Unidade Acadêmica de Garanhuns pela concessão de recursos laboratoriais e pessoais e à Coordenação de aperfeiçoamento de pessoal de nível superior pela fomentação financeira.

\section{REFERÊNCIAS}

1. Anand, T.; Bhaskaran, R.; Karthikeyan, T.R.G.M.R.; Senthilraja, G. Production of cell wall degrading enzimes and toxins by Colletotrichum Capsici and Alternaria alternate Causing Fruit rot of Chillies. Journal of Plant Protection, Wielkopolskie, v.48, n.4, p.437-451, 2008.

2. Ângelo, R.S. Enzimas hidrolíticas. In: Esposito, E.; Azevedo, J.L. Fungos: Uma introdução a biologia, bioquímica e biotecnologia. 2. ed. Caxias do Sul: Educs, 2010, p. 245-260.
3. Baer, D.; Gudmestad, N.C. In vitro cellulolytic activity of the plant pathot gen Clavibacter michiganensis subsp. sepedonicus. Canadian Journal of Microbiolology, Ottawa, v.41, n.10, p.877-888. 1995.

4. Basso, T.P.; Gallo, C.R.; Basso, L.C. Atividade celulolítica de fungos isoł lados de bagaço de cana-de-açucar e madeira em decomposição. Pesquisa agropecuária brasileira, Brasília, v.45, n.11, p.1282-1289. 2010.

5. Bradford, M.M.A Rapid and Sensitive Method for the Quantitation of Microgram Quantities of Protein Utilizing the Principle of Protein-Dye Binding. Analytical Biochemistry, Bethesda, n.72, p.248-254. 1976.

6. Carlile, M.J.; Watkinson, S.C.; Gooday, C.H. The fungi. London: Academic Press, 2001. 588p.

7. Castro, A.M.; Pereira Júnior, N. Produção, propriedade e aplicação das celulases na hidrólise de resíduos agroindustriais. Química Nova, São Paulo, v.33, n.7, p.181-188. 2010.

8. Deshpande, V.; Eriksson, K.E. 1,4- $\beta$-D-glucosidases of Sporotrichum pulverulentum. In: Wood, W. A. e Kellogg, S.T. Methods in Enzymology. San Diego: Academic Press, 1988, p. 415-424.

9. Ghose, T.K. Measurement of Cellulase Activities. Pure and Applied Chemistry, Switzerland, v.59, n.2, p.257-268, 1987.

10. Kikot, G.E.; Hours, R.A.; Alconada, T.M. Extracellular Enzymes of Fusarium graminearum Isolates. Brazilian Archives of Biology and Technology, Curitiba, v.53, n.4, p.779-783, 2010.

11. Lima Filho, R.M.; Oliveira, S.M.A.; Menezes, M. Caracterização enzimática e patogenicidade cruzada de Colletotrichum spp. associados a doenças de pós-colheita. Fitopatologia Brasileira, Brasília-DF, v.28, n.6, p.620-625, 2003.

12. Liming, X.; Xueliang, S. High-yeld production by Trichoderma reesei ZU-02 on corn cob residue. Bioresource Technology, Amsterdam, v.91, n.3, p.259-262, 2004

13. Lynd, L.R.; Weimer, P.J.; Vanzil, W.H.; Pretorius, I.S. Microbial Cellulose Utilization. Fundamentals and Molecular Biology Rewiews, New York, v.66, n.3, p.506-577, 2002.

14. Malik, S.K.; Mukhtari, H.; Farooqi, A.A.; Ikram-ul-haq, M. Optimization of process parameters for the biosynthesis of cellulases by Trichoderma viride. Pakistan Journal of Botany, Karachi, v.42, n.6, p.4243-4251, 2010

15. Massola, N.S.; Bedendo, I.P. Doenças da Mandioca. In: Kimati, H.; Amorim, L.; Rezende, J.A.M.; Bergamin Filho, A.; Camargo, L.E.A. Manual de Fitopatologia: Doenças das Plantas Cultivadas. São Paulo: Ceres, 2005. v.2, p.449-455.

16. Mendgen, K.M.; Hahn, H. Deising.Morphogenesis and Mechanisms of Penetration by Plant Pathogenic Fungi. Annual Review of Phytopathology, Palo Alto, n.34, p.364-386, 1996.

17. Moldoveanu, N.; Kluepfel, D. Comparison of $\beta$-Glucosidase Activities in Different Streptomyces Strains. Applied and Environmental Microbiology, Washington, v.46, n.1, p.17-21. 1983

18. Morais, M.S.; Nascimento, L.C.; Moreira, K.A.; Cavalcanti, M.S. e Oliveira, N.T. Levantamento e avaliação da incidência das doenças da mandioca no estado da Paraíba. Summa Phytopathologica, Botucatu, v.39, n.3, p.204-206, 2013.

19. Moreira, F.G.; Reis, S.; Costa, M.A.F.; Souza, C. G. M.; Peralta, R.M. Production of Hydrolytic Enzimes by the Plant Pathogenic Fungus Myrothecium verrucaria in Submerged Cultures. Brazilian Journal of Microbiology, São Paulo, v.36, n.1, p.7-11, 2005

20. Narasimha, G.; Sridevi, A.; Buddolla, V.; Subhosh C.M.; Rajasekhar, R.B. Nutrient effects on production of cellulolytic enzymes by Aspergillus niger. African Journal of Biotechnology, Lagos, v.5, n.5, p.472-476, 2006.

21. Okunowo, W.O.; Gbenle, G.O.; Osuntoki, A.A.; Adekunle, A.A. e Ojokuku, S.A. Production of cellulolytic and xylanolytic enzymes by a phytopathogenic Myrothecium roridum and some avirulent fungal isolates from water hyacinth. African Journal of Biotechnology, Lagos, v.7, n.9, p.1074-1078, 2010.

22. Park, E.Y.; Naruse, K. e Tatsuya, K. Improvement of cellulase production in cultures of Acremonium cellulolyticus using pretreated waste milk pack with cellulase targeting for biorefinery. Bioresource Technology, Amsterdam, v.102, n.10, p.6120-6127, 2011.

23. Pascholati, S.F. Fitopatógenos: Arsenal Enzimático. In: Bergamin filho, A.; Kimati, H.; Amorim, L. Manual de Fitopatologia: Princípios e Conceitos. São Paulo: Ceres, 1995. v.1, p.343-364.

24. Paterson, R.R.M.; Bridge, R.L.D.L. Biochemical techniques for filamentous fungi. Cambridge: CAB International, 1994. 125p.

24. Ramanathan, G.; Banupriya, S.; Abirami, D. Production and Optimization of 
Cellulase from Fusarium oxysporum by submerged fermentation. Journal of Scientific \& Industrial research, New Delhi, n.69, p.454-459, 2010.

25. Senaratna, L.K.; Wijesundera R.L.C.; Liyanage, A.S. Morphological and physiological characters of two isolates of Colletotrichum gloeosporioides from rubber (Hevea brasiliensis). Mycological Research, Manchester, v.95, n.9, p.1085-1089, 1991.

26. Singh, A.; Singh, N.; Bishnoi, N.R. Production of Cellulases by Aspergillus Heteromorphus from wheat straw under submerged fermentation. International Journal of Civil and Environmental Engineering, Saddar, v.1, n.1, p.23-26, 2009.

27. Sukumaran, R.K.; Singhania, R.R.; Pandey, A. Microbial cellulases -production, applications and challenges. Journal of Science Industrial and Research, New Delhi, v.64, n.11, p.832-844, 2005.

28. Talekhar, S.; Ghodake, V.; Chavare, S. Production and characterization of Cellulase by local fungal isolate of India using water hyacinth as carbon source and reuse of fungal biomass for dye degradation. International Journal of Engineering Science and Technology, Otteri, v.3, n.4, p.3236$3241,2011$.

29. Wen, Z.; Liao, W.; Chen, S. Production of cellulase//-glucosidase by the mixed fungi culture of Trichoderma reesei and Aspergillus phoenicis on dairy manure. Applied Biochemistry Biotechnology, Hoboken, v.121, n. 1, p.93-104, 2005 